九州大学学術情報リポジトリ

Kyushu University Institutional Repository

\title{
Metabolomic profiling of brain tissues of mice chronically exposed to heroin
}

Li, Ren-Shi

Laboratory of Molecular Life Sciences, Graduate School of Pharmaceutical Sciences, Kyushu University

Takeda, Tomoki

Laboratory of Molecular Life Sciences, Graduate School of Pharmaceutical Sciences, Kyushu University

Ohshima, Takashi

Green Pharmaceutical Chemistry, Graduate School of Pharmaceutical Sciences, Kyushu University

Yamada, Hideyuki

Laboratory of Molecular Life Sciences, Graduate School of Pharmaceutical Sciences, Kyushu University

他

http://hdl. hand le. net/2324/1808309

出版情報：Drug Metabolism and Pharmacokinetics. 32 (1)，pp.108-111，2016-10-27. Elsevier バージョン：

権利関係 : 
Metabolomic profiling of brain tissues of mice chronically exposed to heroin

Ren-Shi Li ${ }^{\text {a }}$, Tomoki Takeda ${ }^{\text {a }}$, Takashi Ohshima ${ }^{b}$, the late Hideyuki Yamada ${ }^{\text {a }}$, Yuji Ishii ${ }^{a *}$

${ }^{a}$ Laboratory of Molecular Life Sciences, and ${ }^{b}$ Green Pharmaceutical Chemistry,

Graduate School of Pharmaceutical Sciences, Kyushu University; 3-1-1 Maidashi,

Higashi-ku, Fukuoka 812-8582, Japan

*Corresponding author

Phone: $+81-92-642-6586$.

FAX: $+81-92-642-6588$.

E-mail: ishii@phar.kyushu-u.ac.jp 


\section{ABSTRACT}

The chronic neurotoxicity of heroin on the nervous system is poorly understood. To address this issue, we comprehensively assessed the alteration of brain metabolomics caused by chronic heroin exposure and the withdrawal of heroin. Male C57BL/6J mice $(n=10)$ were given heroin $(15 \mu \mathrm{mol} / \mathrm{kg}$, i.p., twice a day) for 12 days while the withdrawal group received saline-treatment instead of heroin for the last two days. The control group received saline. We developed an UPLC-TOF/MS-based metabolomic approach to analyze the metabolites and carry out a metabolic pathway analysis in the brain. The major metabolites contributing to the discrimination were identified as amino acids, tricarboxylic-acid cycle intermediates, neurotransmitters, nucleotides and other compounds. A marked reduction in histidine and a slight but significant increase in phenylalanine and tryptophan were observed after heroin was withdrawn while the increased level of catecholamines was restored to baseline. Interestingly, $\mathrm{N}$-acetylserotonin - a precursor of melatonin - was increased with the withdrawal of heroin while melatonin was markedly reduced along with the sub-chronic exposure to heroin. This shows that heroin disrupts not only the energy metabolism but also the biosynthesis of both catecholamines and melatonin in the mouse brain. Therefore, these substances are candidate biomarkers for chronic heroin-abuse.

Keywords: metabolomics; metabolic effect; heroin abuse; neurotransmitter;

\section{UPLC-TOF/MS}




\section{Introduction}

Heroin is the most widely abused opioid in the world [1]. It can lead to addiction quickly and does great harm to individuals, both physiologically and psychologically [2]. It can be rapidly metabolized to morphine and other metabolites, which can bind to opioid receptors in the brain [3]. Clearly, drug-induced stable changes in the brain at the molecular level would produce addictive behavior. Repeated heroin use changes the physical structure and physiology of the brain [4], creating long-term imbalances in neuronal and hormonal systems which are not easily reversed $[5,6]$.

Metabolomics aims to extract, separate and analyze the totality of small molecules present in any biological system (such as biofluids and tissues) or any specific physiological state in an effective and reproducible way [7]. It has been widely applied in toxicological studies, especially when investigating the action mechanism of toxicity $[8,9]$. Furthermore, because heroin can be rapidly metabolized in vivo, heroin itself cannot be used as the target analyte to identify heroin abuse in practice. Therefore, we use endogenous compounds as metabolic markers to identify heroin abuse and this is an alternative approach, which can also reveal the underlying mechanisms.

Previous metabolomics studies have shown metabolic alterations in rats exposed to heroin using blood and urine samples [2]. However, the effect of heroin on the brain metabolome has not been clarified and the sub-chronic neurotoxicity of heroin at low 
levels in the brain is poorly understood. The purpose of this study was to identify the potential brain biomarkers and the molecular neurotoxicity mechanisms in mice exposed to heroin and following heroin withdrawal.

\section{Materials and methods}

\subsection{Chemicals and reagents}

Heroin hydrochloride was synthesized by acetylation of the two-hydroxyl groups of morphine with acetic anhydride in our laboratory [10]. All other reagents were of the highest grade commercially available.

\subsection{Animal treatment and sample collection}

Male C57BL/6J mice (20-25g) were purchased from CLEA Japan, Inc (Tokyo, Japan). The mice were housed in a temperature-controlled environment $\left(20 \pm 4^{\circ} \mathrm{C}\right)$, with a $12 \mathrm{~h}$ light-dark cycle (lights on at 7: 00) and a relative humidity of $50 \pm 20 \%$, with free access to food and water. After adaptation to standard laboratory conditions for 1 week, the mice were randomly allocated to 3 groups ( $n=10 /$ group): a heroin-treated group, a heroin-withdrawal group and a control group. All animal experiments were conducted with the approval of the Institutional Animal Care and Experiment Committee of Kyushu University.

Heroin hydrochloride was dissolved in saline on the day of use and all mice were weighed every day prior to administration. The mice in the heroin-treated group received an i.p. injection of heroin $(15 \mu \mathrm{mol} / \mathrm{kg})$ twice a day $(9$ am and $5 \mathrm{pm}$, 
respectively) for 12 consecutive days. The dose used was chosen according to the published studies $[11,12]$. The mice in the heroin-withdrawal group received an i.p. injection of heroin $(15 \mu \mathrm{mol} / \mathrm{kg})$ twice a day for 10 consecutive days followed by withdrawal of heroin for 2 days. The mice in the control group received an i.p. injection of saline for 12 days. Then, the mice were anesthetized by $\mathrm{CO}_{2}$ inhalation and their brains were rapidly removed, and rinsed with saline. Finally, the brain tissues were transferred to $1.5 \mathrm{ml}$ polypropylene tubes, snap-frozen in liquid nitrogen and stored at $-80^{\circ} \mathrm{C}$ until analysis.

A liquid-liquid extraction method was used for metabolite extraction from each brain tissue sample [13]. The residue was finally reconstituted in $100 \mu 1 \mathrm{CH}_{3} \mathrm{CN} / \mathrm{H}_{2} \mathrm{O}$ $(1: 1, \mathrm{v} / \mathrm{v})$, and an aliquot $(10 \mu \mathrm{l})$ of the solution was subjected to analysis by UPLC-TOF/MS (see the Supplemental Methods S1 for the detailed conditions).

\subsection{Data processing and metabolite identification}

Data processing was performed using MassLynx v4.1 and MarkerLynx XS v4.1 software (Waters Co., Milford, MA). To compare metabolomic profiles between the control and heroin-treated group or between the control and heroin-withdrawal group, the data were then analyzed by an orthogonal partial least squares-discriminant analysis (OPLS-DA) and S-plot method using MarkerLynx XS v4.1 software [14, 15]. (see the Supplemental Methods S2 for the detailed conditions).

\section{Results and discussion}

After heroin administration, the mice became excited, nervous and very sensitive to 
disturbances. Subsequently, after being excited for approximately 1 hour, the mice gradually became calm. In addition, there was a reduction in body weight immediately after heroin administration (see supplemental Fig. S1).

We subsequently used a multivariate analysis method with UPLC-MS data to make metabolite variations prominent and to identify the metabolites responsible for such variations. The corresponding score plots from the supervised OPLS-DA showed a clear difference between the two groups (Fig. 1). The S-plot, which was derived from the OPLS-DA, was used to select potential biomarkers and visualize metabolomics changes. The farthest red dots were the most likely potential biomarkers because of their high contributions and correlations. A list of the identified biomarkers is given in Table 1. The intensities of the peaks obtained from MarkerLynx were used to calculate the relative concentrations of the biomarkers.

Some selected biomarkers were further investigated. These reflect the metabolic responses of the mice to heroin exposure and can reveal potential markers of metabolic perturbation and provide data for exploring the underlying mechanisms of action. Citrate is a crucial intermediate of the tricarboxylic acid (TCA) cycle [2]. Elevation of citrate indicates up-regulation of the TCA cycle. It has been reported that loss of appetite is one of the symptoms of heroin addiction [16]. On the other hand, in response to heroin administration, mice consumed more energy source materials (Table 1). Since it is known that body weight loss is also associated with energy consumption $[2,17]$, the heroin-induced effect was probably associated with similar mechanisms. Nucleotide monophosphates, which are metabolites of nucleotide 
triphosphates, were increased significantly in the brain following heroin treatment. It is generally believed that nucleotide metabolomics can provide much energy, resulting in increased production of nucleotide monophosphates [18]. Glutamate is an important excitatory neurotransmitter that plays the principal role in central nervous system (CNS) [19]. Elevation of glutamate suggested excitation of the animals by chronic heroin exposure.

Furthermore, heroin can lead to autonomic nervous-system dysfunction and neurotoxicity. Catecholamines in the brain are thought to play a role in mood regulation [20]. In our study, tyrosine was reduced after heroin administration. In CNS, there are many kinds of important neurotransmitters related to the metabolism of tyrosine; e.g. dopamine, adrenaline and noradrenaline. The metabolic pathway of tyrosine in the CNS is shown in Fig. S2. These peripheral metabolites were identified as potential surrogate markers characterizing the metabolic effect of heroin on CNS function. Although both dopamine and adrenaline were elevated by chronic heroin exposure, the effect on the level of adrenaline was far lower than that for dopamine, whereas the level of these catecholamines returned to baseline after the withdrawal of heroin for 2 days. The significant increase in dopamine might be characteristic of heroin-induced toxicity in the brain [21]. Further studies are necessary to elucidate the mechanism underlying the complex modulation of catecholamines by heroin-exposure. These catecholamines may be indirect biomarkers and can not only be used to understand the whole system, but also reveal new insights into the mechanism of toxicity. Interestingly, phenylalanine, a precursor of catecholamine showed a slight 
but significant increase in the withdrawal group. Furthermore, heroin produces an elevation of histamine while a marked decrease in histidine was observed after heroin withdrawal. This agrees with the report that morphine increases the release of histamine from mast-cells [22]. However, the mechanism for the reduction in histidine following heroine withdrawal remains to be elucidated. Tryptophan and $\mathrm{N}$-acetylserotonin, precursors of melatonin, were increased by heroin-withdrawal while melatonin was markedly reduced by sub-chronic exposure to heroin. Melatonin supplementation is known to be associated with the syndrome elicited by heroin withdrawal [23]. Therefore, a reduction of melatonin is a possible biomarker of heroin abuse while an increase in tryptophan and $\mathrm{N}$-acetylserotonin are possible biomarkers of heroin withdrawal. Further studies are necessary to clarify the effect on the levels of mRNA and protein expression of genes related to these pathways.

\section{Conclusions}

Metabolomics, as a systemic toxicological approach, is able to provide comprehensive information about heroin-induced toxicity. Metabolic profiling can aid the mechanistic elucidation of toxicological and pathologic changes and can be a means of identifying potential biochemical markers of toxicity. We have demonstrated that our metabolomics method based on UPLC-TOF/MS can be successfully applied to profile biomarkers and help us understand the molecular mechanisms of heroin abuse. 


\section{Acknowledgments}

This study was supported in part by a Health and Labour Sciences Research Grant

from the Ministry of Health, Labour and Welfare of Japan (H27-Designated Research-003).

\section{Supplementary information}

Supplementary data associated with this article can be found, in the online version.

\section{Conflict of interest}

None of the authors has any conflict of interest to disclose.

\section{References}

[1] Caplan LR, Hier DB, Banks G. Current concepts of cerebrovascular disease--stroke: stroke and drug abuse. Stroke 1982;13:869-872.

[2] Zheng T, Liu L, Aa J, Wang G, Cao B, Li M, et al. Metabolic phenotype of rats exposed to heroin and potential markers of heroin abuse. Drug Alcohol Dependence 2013;127:177-186.

[3] Gottås A, Øiestad EL, Boix F, Vindenes V, Ripel A, Thaulow CH, and Mørland J. Levels of heroin and its metabolites in blood and brain extracellular fluid after i.v. heroin administration to freely moving rats. Br J Pharmacol 2013;170:546-556.

[4] Wang X, Li B, Zhou X, Liao Y, Tang J, Liu T, Hu D, Hao W. Changes in brain gray matter in abstinent heroin addicts. Drug Alcohol Depend 2012;126:304-308.

[5] Ignar DM, Kuhn CM. Effects of specific mu and kappa opiate tolerance and abstinence on hypothalamo-pituitary-adrenal axis secretion in the rat. J Pharmacol 
Exp Ther 1990;255:1287-1295.

[6] Kreek MJ, Ragunath J, Plevy S, Hamer D, Schneider B, Hartman N. ACTH, cortisol and beta-endorphin response to metyrapone testing during chronic methadone maintenance treatment in humans. Neuropeptides 1984;5:277-278.

[7] Griffiths WJ, Koal T, Wang Y, Kohl M, Enot DP, Deigner HP. Angew.Chem. Int. Ed. Targeted metabolomics for biomarker discovery 2010;49:5426-5445.

[8] Zaitsu K, Hayashi Y, Suzuki K, Nakayama H, Hattori N, Takahara R, Kusano M, Tsuchihashi H, Ishii A. Metabolome disruption of the rat cerebrum induced by the acute toxic effects of the synthetic cannabinoid MAM-2201. Life Sci 2015;137:49-55

[9] Zaitsu K, Hayashi Y, Kusano M, Tsuchihashi H, Ishii A. Application of metabolomics to toxicology of drugs of abuse: A mini review of metabolomics approach to acute and chronic toxicity studies. Drug Metab Pharmacokinet 2016;31:21-26.

[10] Cooper GK , Rapoport H. Synthesis of N-p-azidophenylethyl-7,8-dihydronormorphine and its 7,8-ditritio analogue. Potential opiate receptor photoaffinity labels. J Label Compd Radiopharm 1985;22:1201-1207. [11] Karinen R, Andersen JM, Ripel A, Hasvold I, Hopen AB, Mørland J, Christophersen AS. Determination of Heroin and Its Main Metabolites in Small Sample Volumes of Whole Blood and Brain Tissue by Reversed-Phase Liquid Chromatography-Tandem Mass Spectrometry. J Anal Toxicol 2009;33:345-350.

[12] Andersen JM, Ripel A, Boix F, Normann PT, Mørland J. Increased locomotor activity induced by heroin in mice: pharmacokinetic demonstration of heroin acting as 
a prodrug for the mediator 6-monoacetylmorphine in vivo. J Pharmacol Exp Ther 2009;331:153-161.

[13] Ivanisevic J, Zhu ZJ, Plate L, Tautenhahn R, Chen S, O'Brien PJ, Johnson CH, Marletta MA, Patti GJ, Siuzdak G. Toward 'Omic' scale metabolite profiling: a dual separation-mass spectrometry approach for coverage of lipids and central carbon metabolism. Anal Chem 2013;85:6876-6884.

[14] Trygg J, Holmes E, Lundstedt T. Chemometrics in metabonomics. J Proteome Res 2007;6:469-479.

[15] Jandrić Z, Roberts D, Rathor MN, Abrahim A, Islam M, Cannavan A. Assessment of fruit juice authenticity using UPLC-QToF MS: a metabolomics approach. Food Chem 2014;148:7-17.

[16] Morton GJ, Cummings DE, Baskin DG, Barsh GS, Schwartz MW. Central nervous system control of food intake and body weight. Nature 2006 ;443:289-295.

[17] Leibel RL, Rosenbaum M, Hirsch J. Changes in energy expenditure resulting from altered body weight. N Engl J Med 1995;332:621-628.

[18] Lane AN, Fan TW. Regulation of mammalian nucleotide metabolism and biosynthesis. Nucleic Acids Res 2015;43:2466-2485.

[19] Meldrum BS. Glutamate as a neurotransmitter in the brain: review of physiology and pathology. J Nutr 2000;130:1007S-1015S.

[20] Fenli S, Feng W, Ronghua Z, Huande L. Biochemical mechanism studies of venlafaxine by metabonomic method in rat model of depression. Eur Rev Med Pharmacol Sci 2013;17:41-48. 
[21] Commins DL, Vosmer G, Virus RM, Woolverton WL, Schuster CR, Seiden LS. Biochemical and histological evidence that methylenedioxymethylamphetamine (MDMA) is toxic to neurons in the rat brain. $J$ Pharmacol Exp Ther $1987 ; 241: 338-345$.

[22] Withington DE, Patrick JA, Reynolds F. Histamine release by morphine and diamorphine in man. Anaesthesia 1993;48:26-29.

[23] Cemek M, Büyükokuroğlu ME, Hazman Ö, Bulut S, Konuk M, Birdane Y. Antioxidant enzyme and element status in heroin addiction or heroin withdrawal in rats: effect of melatonin and vitamin E plus Se. Biol Trace Elem Res 2011;139:41-54. 


\section{Figure legends}

Figure 1 Metabolomic profiling of the heroin-treated group or heroin withdrawal group compared with the control group using the UPLC-TOF/MS system $(\mathrm{n}=10 /$ group $)$.

Panels A and C represent the OPLS and S-plot mode of the control vs heroin group. Panels B and D represent the OPLS and S-plot mode of the control vs heroin-withdrawal group. Fragment ions with correlation coefficients of more than +0.8 and less than -0.8 are shown as red dots (panels $\mathrm{C}$ and $\mathrm{D})$. 

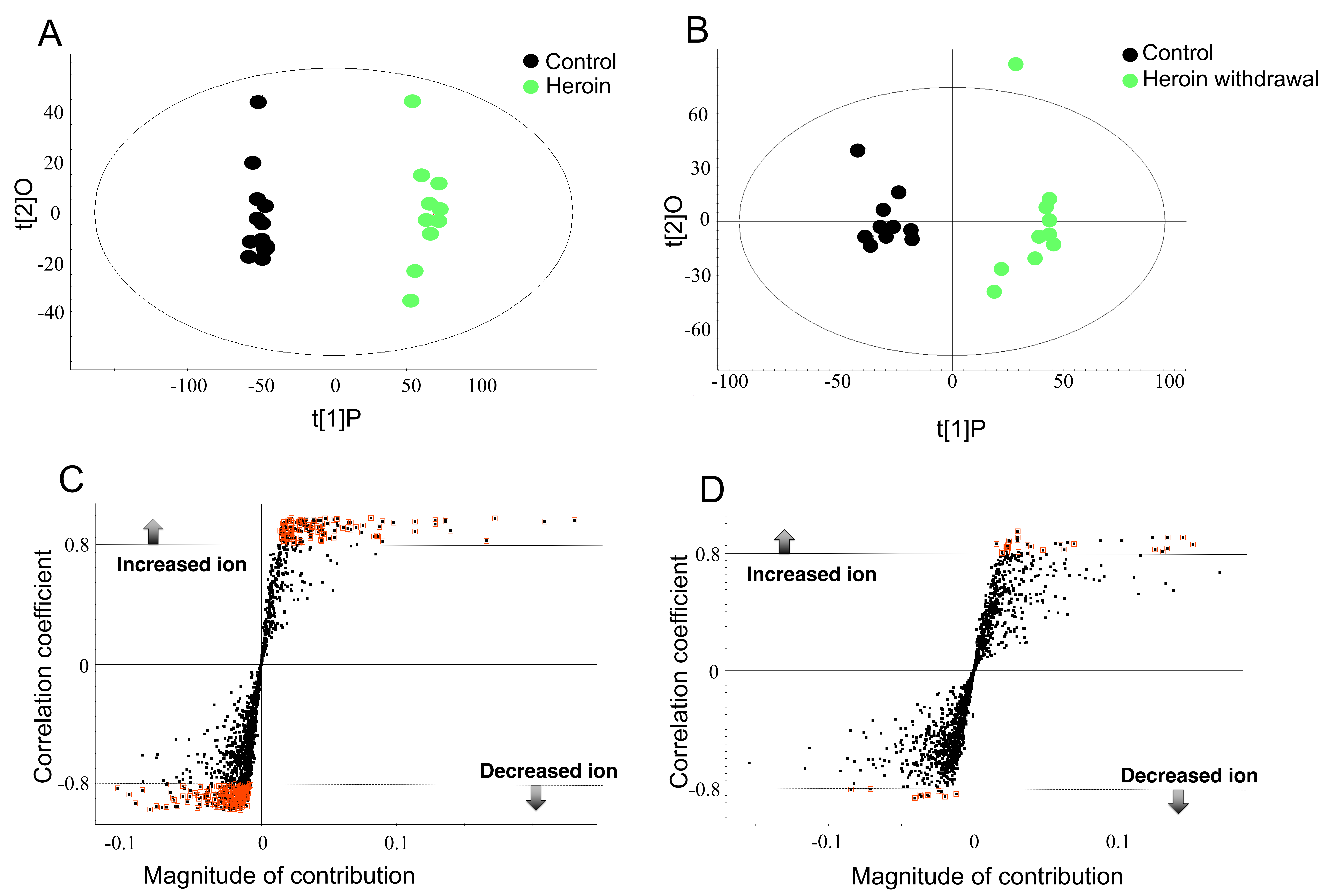

Li et al, Fig. 1 
Table 1 Representative components which were altered by chronic heroin-treatment or heroin-withdrawal: alteration observed in Principal Component Analysis of a change in the profile of brain metabolomes.

\begin{tabular}{|c|c|c|c|c|c|c|c|}
\hline $\begin{array}{l}\text { Metabolic } \\
\text { pathways }\end{array}$ & $\begin{array}{l}\text { Retention } \\
\text { time (min) }\end{array}$ & $\mathrm{m} / \mathrm{z}$ & Metabolite & $\begin{array}{l}\mathrm{x} \text {-Fold } \\
\text { change }^{\mathrm{b}}\end{array}$ & $\begin{array}{c}\mathrm{P} \\
\text { value }\end{array}$ & Trend $^{\mathrm{c}}$ & Treatment \\
\hline \multirow{6}{*}{ Amino acids } & 1.07 & 182.0794 & tyrosine & 2.4 & $8.09 \times 10^{-5}$ & $\downarrow$ & heroin \\
\hline & 21.10 & 148.0651 & glutamate & 1.6 & $4.85 \times 10^{-2}$ & $\uparrow$ & heroin \\
\hline & 1.71 & 150.0638 & methionine & 4.5 & $6.04 \times 10^{-5}$ & $\downarrow$ & heroin \\
\hline & 6.16 & 166.0900 & phenylalanine & 1.5 & $4.08 \times 10^{-5}$ & $\uparrow$ & $\begin{array}{c}\text { heroin } \\
\text { withdrawal }\end{array}$ \\
\hline & 10.88 & 205.0960 & tryptophan & 1.3 & $1.98 \times 10^{-4}$ & $\uparrow$ & $\begin{array}{c}\text { heroin } \\
\text { withdrawal }\end{array}$ \\
\hline & 11.50 & 156.0856 & histidine & $>50$ & $1.08 \times 10^{-4}$ & $\downarrow$ & $\begin{array}{c}\text { heroin } \\
\text { withdrawal }\end{array}$ \\
\hline $\begin{array}{c}\text { TCA } \\
\text { intermediates }\end{array}$ & 16.17 & 193.1709 & citrate & 1.3 & $1.00 \times 10^{-2}$ & $\uparrow$ & heroin \\
\hline \multirow{6}{*}{ Neurotransmitters } & 10.87 & 170.0735 & noradrenaline & 2.7 & $2.00 \times 10^{-7}$ & $\downarrow$ & heroin \\
\hline & 1.17 & 184.0949 & adrenaline & 2.6 & $1.40 \times 10^{-4}$ & $\uparrow$ & heroin \\
\hline & 1.21 & 198.1132 & metanephrine & 5.2 & $3.48 \times 10^{-4}$ & $\uparrow$ & heroin \\
\hline & 1.39 & 154.0796 & dopamine & 18.3 & $1.50 \times 10^{-4}$ & $\uparrow$ & heroin \\
\hline & 12.94 & 161.1111 & tryptamine & 1.9 & $7.22 \times 10^{-4}$ & $\uparrow$ & heroin \\
\hline & 19.97 & 112.0970 & histamine & 2.5 & $8.09 \times 10^{-5}$ & $\uparrow$ & heroin \\
\hline \multirow[t]{2}{*}{ Nucleotides } & 1.27 & 365.0435 & $\mathrm{XMP}^{\mathrm{a}}$ & 4.4 & $1.32 \times 10^{-5}$ & $\uparrow$ & heroin \\
\hline & 1.18 & 371.0426 & OMP & 2.0 & $1.21 \times 10^{-3}$ & $\uparrow$ & heroin \\
\hline \multirow[t]{9}{*}{ Others } & 11.22 & 261.1408 & $\begin{array}{l}\text { glucose-6- } \\
\text { phosphate }\end{array}$ & 7.8 & $2.59 \times 10^{-4}$ & $\downarrow$ & heroin \\
\hline & 4.20 & 269.0931 & inosine & 4.1 & $7.00 \times 10^{-7}$ & $\uparrow$ & heroin \\
\hline & 1.47 & 132.0800 & creatine & $>50$ & $1.00 \times 10^{-7}$ & $\uparrow$ & heroin \\
\hline & 1.71 & 123.0599 & nicotinamide & $>50$ & $1.23 \times 10^{-4}$ & $\downarrow$ & heroin \\
\hline & 20.13 & 152.0543 & guanine & 2.6 & $7.00 \times 10^{-7}$ & $\uparrow$ & heroin \\
\hline & 1.62 & 233.1275 & melatonin & $>50$ & $7.80 \times 10^{-5}$ & $\downarrow$ & heroin \\
\hline & 3.80 & 268.1074 & adenosine & 2.5 & $3.17 \times 10^{-5}$ & $\uparrow$ & heroin \\
\hline & 10.91 & 136.0715 & adenine & 2.0 & 0.205 & $\uparrow$ & $\begin{array}{c}\text { heroin } \\
\text { withdrawal }\end{array}$ \\
\hline & 2.06 & 219.1193 & $\mathrm{~N}$-acetylserotonin & 3.8 & 0.01 & $\uparrow$ & $\begin{array}{c}\text { heroin } \\
\text { withdrawal }\end{array}$ \\
\hline
\end{tabular}

a Notes: XMP, xanthosine monophosphate; OMP, orotidine monophosphate ;

${ }^{\mathrm{b}} \mathrm{x}$-Fold change $=$ heroin-treatment group or heroin withdrawal-treatment group compared with the control group the metabolites concentration change.

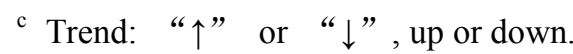


Supporting Information

Metabolomic profiling of brain tissues of mice chronically exposed to heroin

Renshi Li ${ }^{\text {a }}$, Tomoki Takeda ${ }^{\text {a }}$, Takashi Ohshima ${ }^{\text {b }}$, the late Hideyuki Yamada ${ }^{\text {a }}$, Yuji Ishii ${ }^{a *}$

${ }^{a}$ Laboratory of Molecular Life Sciences, and ${ }^{b}$ Green Pharmaceutical Chemistry,

Graduate School of Pharmaceutical Sciences, Kyushu University; 3-1-1 Maidashi,

Higashi-ku, Fukuoka 812-8582, Japan

*Corresponding Author

Phone: $+81-92-642-6586$

Fax: $+81-92-642-6588$

E-mail: ishii@phar.kyushu-u.ac.jp 


\section{Supplemental Methods S1}

\section{Metabolite Extraction}

One $\mathrm{ml}$ of cold $\mathrm{MeOH} / \mathrm{CH}_{3} \mathrm{CN} / \mathrm{H}_{2} \mathrm{O}(2: 2: 1, \mathrm{v} / \mathrm{v})$ solvent was added to $50 \mathrm{mg}$ tissue, vortexed for $30 \mathrm{~s}$ and incubated in liquid nitrogen for $1 \mathrm{~min}$. Samples were allowed to thaw and sonicated for $10 \mathrm{~min}$. This cycle of sample lysis in liquid nitrogen combined with sonication was repeated three times. To precipitate proteins, the samples were incubated for $1 \mathrm{~h}$ at $-20^{\circ} \mathrm{C}$, followed by $15 \mathrm{~min}$ centrifugation at $13,000 \mathrm{rpm}$ and $4^{\circ} \mathrm{C}$. The resulting supernatant was removed and evaporated to dryness. The dry extracts were then reconstituted in $100 \mu \mathrm{CH}_{3} \mathrm{CN} / \mathrm{H}_{2} \mathrm{O}(1: 1, \mathrm{v} / \mathrm{v})$, sonicated for $10 \mathrm{~min}$ and centrifuged for $15 \mathrm{~min}$ at $13,000 \mathrm{rpm}$ and $4^{\circ} \mathrm{C}$ to remove insoluble debris. The supernatants were transferred to UPLC vials and stored at $-80^{\circ} \mathrm{C}$ prior to analysis.

\section{UPLC-TOF/MS conditions}

UPLC-TOF/MS analysis was performed using an ACQUITY UPLC system coupled to a Waters LCT Premier ${ }^{\mathrm{TM}}$ Mass Spectrometer (Waters Corporation, Milford, MA, USA) with electrospray ionization (ESI) in positive and negative modes. A $10 \mu 1$ aliquot of supernatant was injected into an ACQUITY UPLC BEH-C18 column (100 mm $4.6 \mathrm{~mm}$ i.d., $1.7 \mathrm{~mm}$; Waters). For the solvent system, the mobile phases A and B were $0.1 \%$ formic acid in de-ionized water and acetonitrile, respectively. The mobile phase was delivered at a flow rate of $0.2 \mathrm{ml} / \mathrm{min}$, and the entire eluent was passed into a mass spectrometer. The linear gradient was as follows: [\% of B in A (min)]: $2 \%(0$ 8), 2 to $100 \%(8-16), 100 \%$ (16-18), 100 to $2 \%(18-20)$ and $2 \%(20-25)$. A quality 
control (QC) sample was used to optimize the UPLC-TOF/MS conditions, as it contained most of the information of whole samples. For MS analysis, the optimal conditions of analysis were as follows: the source temperature was set at $120^{\circ} \mathrm{C}$ with a cone gas flow of $50 \mathrm{~L} / \mathrm{h}$; a desolvation gas temperature of $350^{\circ} \mathrm{C}$ and a desolvation gas flow of $650 \mathrm{~L} / \mathrm{h}$ were used; the capillary voltage was set at $3000 \mathrm{~V}$ in positive ESI mode, and the cone voltage to $50 \mathrm{~V}$. The MS data were collected in full-scan mode from $\mathrm{m} / \mathrm{z} 50$ to 1000 . For accurate mass acquisition, a lock mass of leucineenkephalin was used via a lock spray interface to ensure accuracy during the MS analysis.

\section{Supplemental Methods S2}

Data processing and metabolite identification

Masslynx v4.1 was used for data acquisition and collection and MarkerLynx XS v4.1 was used for noise filtering, peak detection, alignment of retention time and mass, as well as optional peak normalization. The method parameters for Markerlynx XS v4.1 processing were: peak width at $5 \%$ height, $1 \mathrm{~s}$; peak-to-peak baseline noise, 50 counts; marker intensity threshold, 50 counts; mass window, $0.05 \mathrm{Da}$; retention time window, $0.1 \mathrm{~min}$; and noise elimination level, 6. To compare metabolomic profiles between the control and heroin-treated group or between the control and heroinwithdrawal group, the data were then analyzed by an orthogonal partial least squaresdiscriminant analysis (OPLS-DA) method using MarkerLynx XS v4.1 software. From the S-plot obtained, fragment ions with correlation coefficients of more than +0.8 and 
less than -0.8 were extracted as significantly-increased and -decreased ions by heroin or heroin-withdrawal treatment, respectively. The elemental compositions of the exact masses on the S-plot (Fig. 1) were identified based on the accurate mass and the values of $\mathrm{mDa}$ (the difference from the exact mass) and i-FIT (the correctness of isotope patterns of elemental composition; the lower i-FIT normalized values mean high) of each candidate. The possible compounds with good values of $\mathrm{mDa}$ and i-FIT level were extracted from these prospective formulas in this study. The discovered biomarkers (in Table 1) were extracted from the Masslynx self-bring marker database, the HMDB database (http://hmdb.ca/), the PubChem compound database (http://www.ncbi.nlm.nih. gov), and KEGG (http://www.genome.jp/kegg/ligand.html) using the exact masses of potential biomarkers. The target masses of candidate metabolites identified during the profiling process were searched over a narrow 0.05 Da mass window in the databases as well as the maximum elemental composition of $\mathrm{C}=500, \mathrm{H}=1000, \mathrm{~N}=200, \mathrm{O}=200, \mathrm{~S}=10, \mathrm{P}=10$, and $\mathrm{Cl}=10$. The significance of variations between groups was determined using a $t$-test with Excel 2011 (Microsoft, USA). 


\section{Supplemental Figure S1}

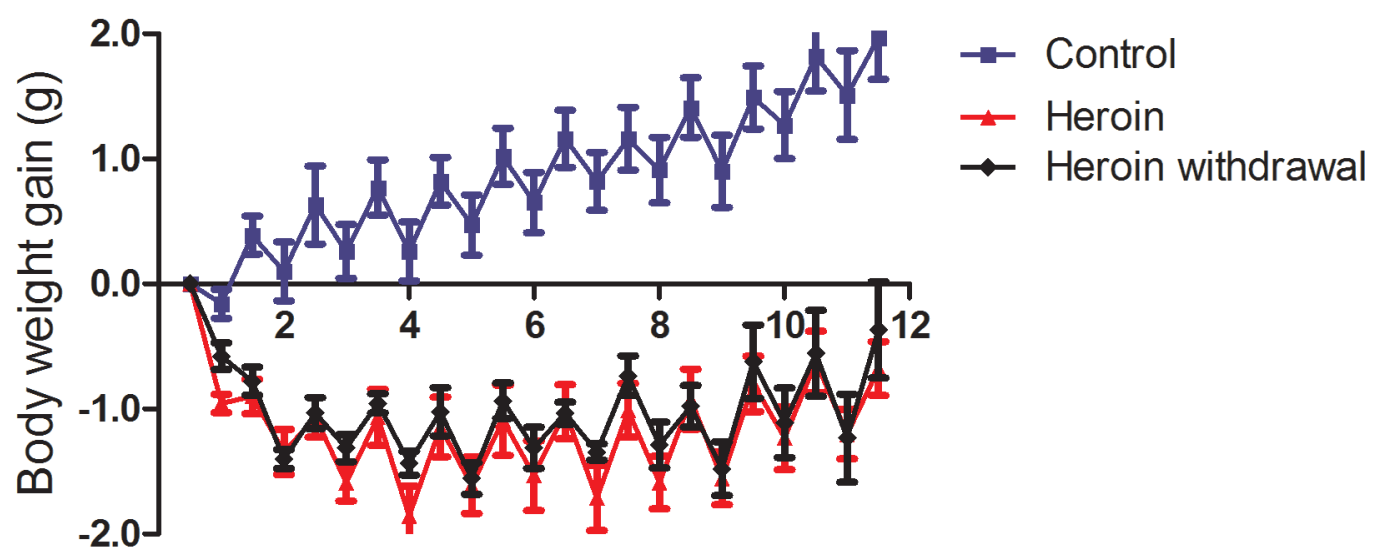

Days after treatments

Fig. S1 Body weight gain of heroin-treated, heroin withdrawal animals and normal controls.

Value represents mean \pm S.E. of ten mice. Samples are the control group (saline, twice a day for 12 days), closed squares (blue) and solid line (blue); heroin-treated group ( $15 \mu \mathrm{mol} / \mathrm{kg}$, twice a day for 12 days), closed triangles (red) and solid line (red); heroin withdrawal group (herointreatment, twice a day for 10 days and then saline twice a day for two days), closed diamonds (black) and solid line (black), respectively. 


\section{Supplemental Fig. S2}<smiles>N[C@@H](Cc1ccc(O)cc1)C(=O)O</smiles>

\section{L-tyrosine}<smiles>N[C@@H](Cc1ccc(O)c(O)c1)C(=O)O</smiles>

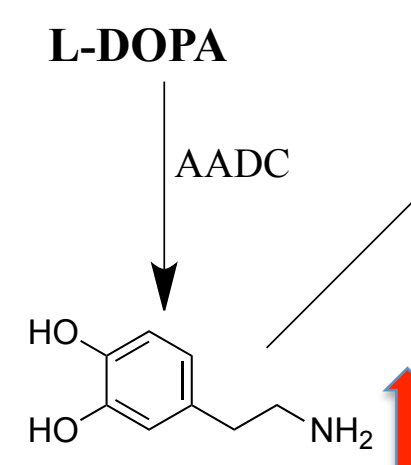

Dopamine

Fig. S2 The effect of heroin on the biosynthesis pathway of catecholamine

Arrows represent the direction of the effect of chronic heroin-treatment.

Abbreviations :

AAAH: Biopterin-dependent aromatic amino acid hydroxylase

AADC: Aromatic L-amino acid decarboxylase

DBH: Dopamine beta-hydroxylase

PNMT: Phenylethanolamine N-methyltransferase 